\title{
El efecto placebo y la ética en la práctica médica
}

\author{
The placebo effect and ethics in medical practice
}

El placebo está incluido dentro de la actividad médica desde hace muchos siglos, siendo que era una palabra en latín, denominada "agradaré". Durante muchos años, la sustancia que generaba el efecto del placebo, le resultaba para muchos médicos que el contenido no era apropiado. No obstante, fueron suponiendo que la acción podría ser convencional, y de esa manera solían mantenerse a través de la costumbre. Luego, varios médicos comenzaron a ver que aunque la sustancia no fuera apropiada, luego de dárselo a los pacientes, ellos podían mejorar por un tratamiento terapéutico. Posteriormente, ciertas investigaciones más recientes han ido demostrando que el efecto del placebo es un evento psicológico y psicofisiológico, por lo tanto, se atribuye que es un contexto terapéutico. Desde hace unos años, también se sabe que la composición principal del placebo es el psicológico, y esto se debe a que las consecuencias son solamente físicas, tal como sucede cuando el dolor desapareció, o ha disminuido. La reducción del dolor por el efecto placebo, es una de sus acciones que más interesa a la comunidad científica, ya que los estudios publicados demuestran que puede sustituir en forma adecuada la acción de los opiáceos. Los placebos, usualmente llamados puros, suelen llevar pastillas de azúcar o una solución salina. y así, el médico lo puede efectuar por sí mismo para tratar de tranquilizar al paciente, o por la petición del propio paciente. Los efectos solo se limitan a aliviar síntomas relativamente superficiales y cuando existe un desequilibrio psicológico, pero no curan totalmente la enfermedad de fondo.

Por lo tanto, el efecto del placebo se señala como psicofisiológico, ya que así, abarca lo físico y asimismo, lo que genera la psicología. Entre los principales resultados psicofisiológicos, el de mayor importancia consiste en la esperanza, luego las expectativas de ciertas respuestas, el poder reducir la ansiedad, la motivación, un cierto condicionamiento, el buen resultado del analgésico, y aquello relativo a lo somático, etc.

La administración de placebos puede promover una mejoría o eventualmente una curación, aunque eso va a depender de la enfermedad, de la personalidad del paciente, y asimismo, de esa capacidad que tenga el médico en sus expectativas al utilizar el placebo.
Los porcentajes del uso del placebo son elevados en las medicinas complementarias y las alternativas (MCA). En EE.UU., en un hospital universitario el $80 \%$ de los adjuntos admitió utilizar ocasionalmente placebos en su práctica clínica, en Dinamarca, un $48 \%$ de los médicos de familia los habían recetado en varias ocasiones a lo largo de un año.

Sin embargo, aún no se define si las MCA son realmente efectivas por sus acciones, $\mathrm{o}$ por lo que suele suponer el paciente mediante sus esperanzas positivas que lo lleva a estar satisfecho, y asimismo, porque mantiene una muy buena relación con el médico que lo escuchó y lo comprendió. Esto ocurre cuando verbalmente los pacientes reciben en forma detallada cuál es el medicamento y sus probables resultados. No obstante, muchas veces la indicación no es correcta, ya sea porque el médico no dice claramente cómo será la acción del medicamento, ni le señala el resultado, y por lo tanto ha manipulado al paciente.

Actualmente, se siguen definiendo diversos aspectos que puedan generar una eficacia real de la MCA, mediante varios estudios que evaluaron científicamente diferentes resultados terapéuticos, en ciertos trastornos y enfermedades. Los estudios que más se han ido estimando en las modalidades pertenecientes a las MCA, han sido evaluadas mayormente por el grupo de la Cochrane Library.

Entre los múltiples estudios que aborda la Cochrane, está la homeopatía, que desde un aspecto científico es algo para discutir sus resultados, ya que está relacionado con la práctica de indicar dosis mínimas, aun en enfermedades graves. Por lo tanto, la medicina no puede señalar si la homeopatía es adecuada en cualquier enfermedad, debido a que es un sistema terapéutico individualizado, y al no evaluarlo en forma apropiada podría generar un trastorno grave en el paciente, debido a que el médico no respeta la ética en su inadecuada conducta.

En relación con los ensayos clínicos aleatorizados, estamos entrando en el terreno de la ética en medicina, porque varias acciones no son correctas. Los ensayos clínicos se suelen dividir en dos grupos de pacientes, uno recibe el medicamento, el otro se denomina grupo control y recibe un placebo. Esta acción en la práctica médica surgió en el siglo 20, y en especial, 
después de la segunda guerra mundial, y en forma paulatina fue aumentando marcadamente. Mediante el contexto de las innovaciones terapéuticas se fue incluyendo la metodología, y así, en esos años fue creciendo la ciencia en la medicina y luego nació la medicina basada en la evidencia. Es lamentable que de a poco, esta medicina fuera invadida por el mercantilismo con sus intereses comerciales inadecuados. Esto fue creciendo enormemente, en especial en algunas industrias farmacéuticas, y en la agobiante tecnología. Es así, que las acciones éticas deben estar siempre presente, ya que el uso inadecuado de los placebos lleva a una actitud no científica y no correcta.

Asimismo, se observa una creciente demanda de la MCA que también plantea algunas cuestiones éticas. Para evaluarlo adecuadamente, se puede contar con diversos aspectos combinados, a través de métodos cualitativos de la investigación, aplicada con la epistemología.

\section{Dr. José María Ceriani Cernadas Editor en Jefe}

http:/ / dx.doi.org/10.5546/ aap.2020.370

Texto completo en inglés:

http: / / dx.doi.org/10.5546/ aap.2020.eng.370

Cómo citar: Ceriani Cernadas JM. El efecto placebo y la ética en la práctica médica. Arch Argent Pediatr 2020;118(6):370-371.

\section{REFERENCIAS}

- Weimer K, Gulewitsch MD, Schlarb AA, Schwille-Kiuntke J, et al. Placebo effects in children: a review. Pediatr Res. 2013;74(1):96-102.

- Grelotti DJ, Kaptchuk TJ. Placebo by proxy. BMJ. 2011; 343:d3435.

- Curie A, Yang K, KirschI, Gollub RL, etal. Placebo Responses in Genetically Determined Intellectual Disability: A metaAnalysis. PLoS One. 2015;10(7):e0133316.

- Bolton P, Shotton L, Young A, Grace J. The ethics of using placebo medication in a non-capacitous patient. Brain Inj. 2012;26(11):1397-400.

- Colloca L, Barsky AJ. Placebo and nocebo effects. N Engl J Med. 2020;382(6):554-61.

- Guerreiro Fregnani JH, Lopes Carvalho A, Rocha F, Souza Viana L, et al. Ética del uso del placebo en la investigación clínica: propuesta de algoritmos para la toma de decisiones. Rev Bioét. 2015; 23(3):456-67.

- Stub T, Foss N, Liodden I. "Placebo effect is probably what we refer to as patient healing power": A qualitative pilot study examining how Norwegian complementary therapists reflect on their practice. BMC Complement Altern Med. 2017;17(1):262. 